\title{
Admissibility Analysis of a Sampled-Data Singular System Based on the Input Delay Approach
}

\author{
Guoquan Chen $\mathbb{D}^{\mathbb{D}}{ }^{1,2}$ Minjie Zheng $\mathbb{D}^{1},{ }^{1}$ Shenhua Yang $\left(\mathbb{D},{ }^{1}\right.$ and Lina Li $\mathbb{D}{ }^{1}$ \\ ${ }^{1}$ Navigation College, Jimei University, Jimei, Xiamen 361021, China \\ ${ }^{2}$ Southampton Business School, University of Southampton, Southampton, SO17 1BJ, UK \\ Correspondence should be addressed to Minjie Zheng; jimi_1205@163.com
}

Received 8 August 2021; Revised 16 December 2021; Accepted 24 January 2022; Published 14 February 2022

Academic Editor: Thach Ngoc Dinh

Copyright (C) 2022 Guoquan Chen et al. This is an open access article distributed under the Creative Commons Attribution License, which permits unrestricted use, distribution, and reproduction in any medium, provided the original work is properly cited.

\begin{abstract}
This study investigates the sampled-data admissibility problem for a singular system. The objective of this paper is to design a sampled-data controller to ensure the admissibility of a singular system and to construct an appropriate Lyapunov-Krasovskii functional (LKF) to get less conservative results for the sampled-data singular system. To accomplish these objectives, the system is converted into a time-delay system by the input delay approach firstly, and both lower and upper bounds of the delay are considered. Secondly, by introducing a suitable LKF, the admissibility criteria are obtained. Then, when estimating the derivative of the LKF, a relaxation variable is introduced by the method of reciprocally convex inequality, and it is proved that the conservatism is reduced. Finally, two numerical examples are given to prove that the designed sampled-data controller can ensure the states of the systems under the influence of external interference.
\end{abstract}

\section{Introduction}

Singular systems have gained considerable attention recently, especially in the research of admissibility and other related theories. Compared with the state-space system, the stability, the regularity, and the impulsivity (or causality) have to be considered in singular systems. The singular system has extensive applications, which cover a variety of engineering fields and practical systems, such as the circuit system, mechanical engineering system, economic system, aerospace system, and robot. Besides, the singular system has more algebraic equations than the normal systems, so the singular system has better extension performance. In recent years, research on the admissibility problem of singular systems has become a hot subject [1-11]. In [7], the admissibility of singular systems is discussed in view of Lyapunov theory, and stability conditions and controller design methods are given. In [8], the robust admissibility issue for the uncertain discrete switched singular system is studied, and the arbitrary switching law and output feedback controller are designed to guarantee that the system is admissible. In [9], the delta operator model for a singular system is obtained by replacing the traditional discrete model with the generalized discrete model. And the relationship between discrete and delta operator models is established. In [10], the admissibility of the stochastic singular system with the T-S fuzzy model is studied, and a new quadratic Lyapunov function is proposed to obtain more relaxed admissibility conditions than the existing methods. The issue about robust admissibility for fuzzy singular systems is studied in [11]. By establishing the LMI, nonquadratic Lyapunov functions are constructed, which achieve less conservative result.

Recently, sampled-data systems have become a hot topic. The system's characteristic is that the discrete and continuous signals are both existing in one system, and it is difficult to be analyzed and designed. Recently, various viewpoints have been reported for sampled-data systems (see [12-20]). The main design methods for analyzing the sampled-data systems can be divided into three kinds. The first one is the lifting technique (see [21]), which converts a continuous system into a discrete system of equal finite dimension. The 
second one is jump system methods (see [22]), where the performance analysis of the sampling system is transformed into the solution of two Riccati equations with mutual jump. However, the two methods mentioned above cannot solve the uncertainty problems caused by the sampling time or system matrix. The third one is the input delay method (see [23]), and the system is transformed into a continuous timevarying delay system, and then the stability analysis can be accomplished using the method of time-delay system. Besides, the input delay approach can deal with the uncertainty of system parameters, and it has been widely used in the sampled-data system (see $[24,25]$ ) and practical engineering field such as autonomous airship, high-speed train, and dynamic positioning ship (see [26-28]). In [24], based on the polytopic linear parameter-varying method, the issue of the sampled-data control is discussed for a nonlinear system. And Wirtinger's inequality is used to obtain less conservative exponential stability condition. In [25], based on the input delay approach, the exponential stability issue for the linear parameter-varying system with aperiodic sampleddata rates is studied. And the distance between actual parameters and measured parameters is considered. In [26], the robust sampled-data control problem of the high-speed train is studied. Through the input delay approach, the highspeed cruise sampled-data controller of the train is designed. It ensures that the train is robust and stable under the interference of external wind. In [27], the sampled-data control issue for an autonomous airship with polyhedral parameter uncertainty is discussed. And a sampled-data controller is designed to guarantee that the system is exponentially stable and meets the $H_{\infty}$ performance index. In [28], a state-derivative control law is provided for a sampleddata dynamic positioning ship. By combining the delaydecomposition technique with Wirtinger's integral inequalities, less conservative results are obtained.

However, although the sampled-data control technology has been well developed in control theory, few results have been reported for the sampled-data singular systems, and the research process on this field is nearly blank. The technical challenges are listed as follows:

(1) The mathematical structure of the sampled-data singular system is more complex

(2) How to improve the LKF to get less conservative results for the sampled-data singular system

(3) How to design a sampled-data controller to guarantee the admissibility of the system and achieve the performance index

As mentioned above, compared with the state-space system, the singular system has richer connotation and wider range. Besides, many multidimensional and multilevel large-scale complex systems are suitable to be dealt with by singular systems. So, it is important and meaningful to analyze the sampled-data control problem for the singular system, which is the motivation of this paper.

In this paper, the admissibility of the sampled-data singular system is studied. Firstly, the system's model is established, and then it is transformed into a time-delay system by using the input delay method. Then, both lower and upper bounds of the sampling period are considered. LMI and Lyapunov functions are introduced for admissibility analysis. By introducing the convex reciprocal inequality, the less conservative results can be obtained. Then, the design method of the sampled-data controller is provided, which makes the system achieve good performance under external interference. Finally, two examples are given to prove the effectiveness of this method.

The main contributions of this article are summarized as follows:

(1) The admissibility condition is established for a sampled-data singular system to ensure the regularity, impulse free, and asymptotical stability. The system has wider application scopes than the existing sampled-data system.

(2) The upper and lower bounds of the variable sampling period are considered, which covers the previous research works as special cases.

(3) Reciprocally convex combination approach is used to estimate the integral terms of the LKF to obtain less conservative results.

Notations: the superscript " $T$ " stands for the transpose. $\mathbb{R}^{m \times n}$ denotes the set of $m \times n$ real matrices. " * "denotes the matrix entries implied by symmetry. $L_{2}[0, \infty)$ is the space of square integrable functions on $[0, \infty)$.

\section{Problem Formulation}

Consider the singular system as follows:

$$
\left\{\begin{array}{l}
E \dot{x}(t)=A x(t)+B u(t)+B_{w} w(t) \\
y(t)=C x(t)+D u(t)
\end{array}\right.
$$

where $x(t) \in \mathbb{R}^{n}$ is the state, $u(t) \in \mathbb{R}^{m}$ is the control input, $y(t) \in \mathbb{R}^{p}$ is the control output, and $w(t) \in \mathbb{R}^{s}$ is the disturbance which belongs to $L_{2}[0, \infty)$ and satisfies that $\int_{0}^{\infty} w^{T}(t) w(t) \mathrm{d} t<\infty$, and it means that the energy of the disturbance is finite. Matrix $E \in \mathbb{R}^{n \times n}$ is assumed to be singular, and rank $(E)=r \leq n . A, B, C, D$, and $B_{w}$ are known constant matrices.

It is assumed that the control signals are obtained at each sampling time $0=t_{0}<t_{1}<\cdots<t_{k}<\cdots<\lim _{k \longrightarrow \infty} t_{k}=+\infty$,
and they have

$$
u(t)=u_{d}\left(t_{k}\right), t_{k} \leq t<t_{k+1}
$$

where $u_{d}$ represents the discrete-time control signal. The proposed sampled-data scheme is depicted in Figure 1.

The sampling period is assumed such that

$$
d_{1} \leq t_{k+1}-t_{k} \leq d_{2}, \quad \forall k \geq 0, d_{2}>d_{1} \geq 0,
$$

where $d_{1}$ and $d_{2}$ are the lower and upper bound of the sampling period. Consider the sampled-data controller as follows:

$$
u(t)=K x\left(t_{k}\right), t_{k} \leq t<t_{k+1},
$$




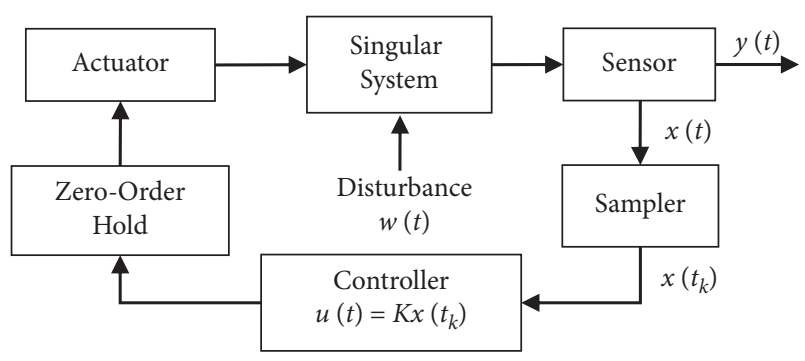

FIgURE 1: The schematic of the sampled-data singular system.

where $K$ is a controller gain matrix. By substituting (4) into (1), then

$$
\left\{\begin{array}{l}
E \dot{x}(t)=A x(t)+B K x\left(t_{k}\right)+B_{w} w(t), t>0, \\
y(t)=C x(t)+D K x\left(t_{k}\right) .
\end{array}\right.
$$

Remark 1. It is noted that both continuous and discrete signals exist in a singular system (5). Therefore, compared with the existing methods for analyzing the continuous or discrete singular systems, it is more complex and meaningful to study the sampled-data control issue for singular systems.

To obtain the main results, we list the following definitions.

\section{Definition 1 (see [29])}

(1) If there exists a constant $s \in \mathbb{C}(\mathbb{C}$ represents the complex field) satisfying $\operatorname{det}(s E-A) \neq 0$, then the matrix pair $(E, A)$ is regular

(2) If $\operatorname{deg}(\operatorname{det}(s E-A))=\operatorname{rank}(E)$, then the matrix pair $(E, A)$ is impulse free

Definition 2 (see [30])

(1) The singular system

$$
E \dot{x}(t)=A x(t)+B x(t-\tau(t))
$$

is said to be regular and impulse free if the pair $(E, A)$ is regular and impulse free.

(2) System (6) is said to be asymptotically admissible if it is regular, impulse free, and asymptotically stable.

The objective of this paper is designing a sampled-data controller to satisfy that

(1) System (5) with $w(t)=0$ is asymptotically admissible

(2) Despite the external disturbances, the output signal $y(t)$ satisfies that $\left\|\left.y(t)\right|_{2} \leq \gamma\right\| w(t) \|_{2}$ for all nonzero $w(t) \in L_{2}[0, \infty)$ under the zero condition, where $\gamma>0$

Based on the input delay approach, define

$$
\tau(t)=t-t_{k}, t_{k} \leq t<t_{k+1},
$$

where the time-varying delay $\tau(t)$ is piecewise linear satisfying $\tau(t) \in\left[d_{1}, d_{2}\right)$. And the derivative of $\tau(t)$ is

$$
\dot{\tau}(t)=1, \quad t \neq t_{k} .
$$

Substituting the time delay $\tau(t)$ into the control input signal $u(t)$, sampled-data controller (4) can be converted such that

$$
\begin{aligned}
u(t) & =u_{d}\left(t_{k}\right)=u\left(t-\left(t-t_{k}\right)\right) \\
& =u(t-\tau(t)) .
\end{aligned}
$$

Substituting (8) into (5), sampled-data system (5) is converted into a singular system with time-varying delay.

$$
\left\{\begin{array}{l}
E \dot{x}(t)=A x(t)+B K x(t-\tau(t))+B_{w} w(t), t>0, \\
y(t)=C x(t)+D K x(t-\tau(t)) .
\end{array}\right.
$$

Remark 2. Compared with $[24,25,31]$ which use the similar input delay approach to deal with the sampleddata problem, it can be seen that if $E=I$, then system (9) is simplified to the normal sampled-data system such as $[24,25,31]$, which shows that the work covers these references as special issues. However, [31] did not consider the variable sampling period and only considered the constant sampling period. $[24,25]$ considered the upper bound of the sampling period, but the lower bound of the sampling period is omitted, which will limit its application scope, and it will also lead to conservatism to some extent. In this paper, the upper and lower bounds of the variable sampling period are considered. Let $d_{1}=d_{2}=d$; the sampling period will reduce to be a constant such as $[24,25,31]$. Thus, the proposed method has wider application scopes than the references. results.

The following lemma will be used for obtaining the main

Lemma 1 (see [32]). For given matrix $J, R=R^{T}>0$, scalars $h_{1}$ and $h_{2}$ satisfying $h_{1} \leq d(t) \leq h_{2}$, and differentiable function $x$ satisfying $\dot{x}:\left[-h_{2},-h_{1}\right] \longrightarrow \mathbb{R}^{n}$, the following inequality holds:

$$
\begin{aligned}
& \left(h_{1}-h_{2}\right) \int_{t-h_{2}}^{t-h_{1}} \dot{x}^{T}(s) R \dot{x}(s) \mathrm{d} s \leq \\
& \leq-\vartheta^{T}(t)\left[\begin{array}{ccc}
I & -I & 0 \\
0 & I & -I
\end{array}\right]^{T} W\left[\begin{array}{ccc}
I & -I & 0 \\
0 & I & -I
\end{array}\right] \vartheta(t),
\end{aligned}
$$

where

$$
\begin{aligned}
\vartheta^{T}(t) & =\left[\begin{array}{lll}
x^{T}\left(t-h_{1}\right) & x^{T}(t-d(t)) & x^{T}\left(t-h_{2}\right)
\end{array}\right], \\
W & =\left[\begin{array}{cc}
-R & J \\
* & -R
\end{array}\right] \leq 0 .
\end{aligned}
$$

\section{Main Results}

In this section, the sufficient admissibility criteria for sampled-data singular systems are exhibited by establishing the LKF and formulating in terms of LMI. 
Theorem 1. For constant delay $d_{1}, d_{2}$, system (9) is asymptotically admissible with $H_{\infty}$ performance $\gamma$ if there exist matrices $P, R, Q_{i}>0, Z_{i}>0, i=1,2,3$, such that

$$
\begin{gathered}
E^{T} P=P^{T} E \geq 0, \\
{\left[\begin{array}{ll}
Z_{3} & R \\
R^{T} & Z_{3}
\end{array}\right]>0,} \\
{\left[\begin{array}{cc}
\Pi_{11} & \Pi_{12} \\
* & \Pi_{22}
\end{array}\right]<0,}
\end{gathered}
$$

where

$$
\begin{aligned}
& \Pi_{11}=\left[\begin{array}{ccccc}
\Xi_{11} & E^{T} Z_{1} E & P^{T} B K & E^{T} Z_{2} E & P^{T} B_{w} \\
* & \Xi_{22} & \Xi_{23} & E^{T} R E & 0 \\
* & * & \Xi_{33} & \Xi_{34} & 0 \\
* & * & * & \Xi_{44} & 0 \\
* & * & * & * & -\gamma^{2} I
\end{array}\right], \\
& \Pi_{12}=\left[\begin{array}{ccccc}
C & 0 & D K & 0 & 0 \\
A & 0 & B K & 0 & B_{w}
\end{array}\right]^{T} \text {, } \\
& \Pi_{22}=\operatorname{diag}\left\{-I,-Z^{-1}\right\} \text {, } \\
& \Xi_{11}=P^{T} A+A^{T} P+Q_{1}+Q_{2}+Q_{3}-E^{T} Z_{1} E-E^{T} Z_{2} E, \\
& \Xi_{22}=-Q_{1}-E^{T} Z_{1} E-E^{T} Z_{3} E, \\
& \Xi_{23}=E^{T} Z_{3} E-E^{T} R E \text {, } \\
& \Xi_{33}=-2 E^{T} Z_{3} E+E^{T} R E+E^{T} R^{T} E, \\
& \Xi_{34}=E^{T} Z_{3} E-E^{T} R E \\
& \Xi_{44}=-Q_{3}-E^{T} Z_{2} E-E^{T} Z_{3} E, \\
& Z=d_{1}^{2} Z_{1}+d_{2}^{2} Z_{2}+d_{21}^{2} Z_{3} \text {, } \\
& d_{21}=d_{2}-d_{1} \text {. }
\end{aligned}
$$

Proof. Firstly, we prove system (9) is regular and impulse free. From (13), it can be obtained that

$$
\begin{gathered}
Q_{1}+Q_{2}+Q_{3}+A^{T} P+P^{T} A-E^{T} Z_{1} E-E^{T} Z_{2} E<0 . \\
\text { As } Q_{1}>0, Q_{2}>0 \text {, and } Q_{3}>0 \text {, then } \\
A^{T} P+P^{T} A-E^{T} Z_{1} E-E^{T} Z_{2} E<0 .
\end{gathered}
$$

Since rank $(E)=r \leq n$, there exist nonsingular matrices $M \in \mathbb{R}^{n \times n}$ and $H \in \mathbb{R}^{n \times n}$ such that

$$
\begin{aligned}
\bar{E} & =M E H \\
& =\left[\begin{array}{ll}
I_{r} & 0 \\
0 & 0
\end{array}\right] .
\end{aligned}
$$

$$
\begin{aligned}
\bar{A} & =M A H \\
& =\left[\begin{array}{ll}
A_{11} & A_{12} \\
A_{21} & A_{22}
\end{array}\right], \\
\bar{P} & =M^{-T} P H \\
& =\left[\begin{array}{ll}
P_{11} & P_{12} \\
P_{21} & P_{22}
\end{array}\right] .
\end{aligned}
$$

From (17) and (18),

$$
\begin{aligned}
\bar{P} & =\left[\begin{array}{cc}
P_{11} & 0 \\
P_{21} & P_{22}
\end{array}\right], \\
P_{11}^{T} & =P_{11}>0 .
\end{aligned}
$$

Premultiplying and postmultiplying $H^{T}$ and $H$ with $\Xi_{11}<0$

$$
A_{22}^{T} P_{22}+P_{22}^{T} A_{22}<0 .
$$

From (20), it means that $A_{22}$ is nonsingular, and the pair $(E, A)$ is regular and impulse free. According to Definition 1, system (9) is regular and impulse free.

Then, we will show that system (9) is asymptotically stable. The LKF is constructed as

$$
\begin{aligned}
V(t)= & \sum_{i=1}^{4} V_{i}(t), t \in\left[t_{k}, t_{k+1}\right) \\
V_{1}(t)= & x(t)^{T} E^{T} P x(t), \\
V_{2}(t)= & \int_{t-d_{1}}^{t} x(s)^{T} Q_{1} x(s) \mathrm{d} s+\int_{t-\tau(t)}^{t} x(s)^{T} Q_{2} x(s) \mathrm{d} s \\
& +\int_{t-d_{2}}^{t} x(s)^{T} Q_{3} x(s) \mathrm{d} s, \\
V_{3}(t)= & d_{1} \int_{-d_{1}}^{0} \int_{t+\theta}^{t} \dot{x}^{T}(s) E^{T} Z_{1} E \dot{x}(s) \mathrm{d} s \mathrm{~d} \theta \\
& +d_{2} \int_{-d_{2}}^{0} \int_{t+\theta}^{t} \dot{x}^{T}(s) E^{T} Z_{2} E \dot{x}(s) \mathrm{d} s \mathrm{~d} \theta, \\
V_{4}(t)= & d_{21} \int_{-d_{2}}^{-d_{1}} \int_{t+\theta}^{t} \dot{x}^{T}(s) E^{T} Z_{3} E \dot{x}(s) \mathrm{d} s \mathrm{~d} \theta .
\end{aligned}
$$

Calculating the derivative of $V(t)$, it can be obtained that

$$
\begin{aligned}
\dot{V}_{1}(t)= & 2 x(t)^{T} E^{T} P \dot{x}(t), \\
\dot{V}_{2}(t)= & x(t)^{T} Q_{1} x(t)-x\left(t-d_{1}\right)^{T} Q_{1} x\left(t-d_{1}\right) \\
& +x(t)^{T} Q_{2} x(t)+x(t)^{T} Q_{3} x(t)-x\left(t-d_{2}\right)^{T} Q_{3} x\left(t-d_{2}\right), \\
\dot{V}_{3}(t)= & d_{1}^{2} \dot{x}(t)^{T} E^{T} Z_{1} E x(t)+d_{2}^{2} \dot{x}(t)^{T} E^{T} Z_{2} E \dot{x}(t) \\
& -d_{1} \int_{t-d_{1}}^{t} \dot{x}^{T}(s) E^{T} Z_{1} E \dot{x}(s) \mathrm{d} s \\
& -d_{2} \int_{t-d_{2}}^{t} \dot{x}^{T}(s) E^{T} Z_{2} E \dot{x}(s) \mathrm{d} s, \\
\dot{V}_{4}(t)= & d_{21}^{2} \dot{x}(t)^{T} E^{T} Z_{3} E \dot{x}(t)-d_{21} \int_{t-d_{2}}^{t-d_{1}} \dot{x}^{T}(s) E^{T} Z_{3} E \dot{x}(s) \mathrm{d} s .
\end{aligned}
$$


According to Jensen's inequality, it can be obtained that

$$
\begin{aligned}
& -d_{1} \int_{t-d_{1}}^{t} \dot{x}^{T}(s) E^{T} Z_{1} E \dot{x}(s) \mathrm{d} s \leq \\
& -\left[x(t)-x\left(t-d_{1}\right)\right]^{T} E^{T} Z_{1} E\left[x(t)-x\left(t-d_{1}\right)\right], \\
& -d_{2} \int_{t-d_{2}}^{t} \dot{x}^{T}(s) E^{T} Z_{2} E \dot{x}(s) \mathrm{d} s \leq \\
& -\left[x(t)-x\left(t-d_{2}\right)\right]^{T} E^{T} Z_{2} E\left[x(t)-x\left(t-d_{2}\right)\right],
\end{aligned}
$$

and

$$
\begin{aligned}
& -d_{21} \int_{t-d_{2}}^{t-d_{1}} \dot{x}^{T}(s) E^{T} Z_{3} E \dot{x}(s) \mathrm{d} s \\
& =-d_{21} \int_{t-d_{2}}^{t-\tau(t)} \dot{x}^{T}(s) E^{T} Z_{3} E \dot{x}(s) \mathrm{d} s \\
& -d_{21} \int_{t-\tau(t)}^{t-d_{1}} \dot{x}^{T}(s) E^{T} Z_{3} E \dot{x}(s) \mathrm{d} s \\
& \leq-\frac{d_{21}}{d_{2}-\tau(t)}\left(x(t-\tau(t))-x\left(t-d_{2}\right)\right)^{T} \\
& \quad \times E^{T} Z_{3} E\left(x(t-\tau(t))-x\left(t-d_{2}\right)\right) \\
& \quad+\frac{d_{21}}{\tau(t)-d_{1}}\left(x\left(t-d_{1}\right)-x(t-\tau(t))\right)^{T} \\
& \quad \times E^{T} Z_{3} E\left(x\left(t-d_{1}\right)-x(t-\tau(t))\right) .
\end{aligned}
$$

It can be found from (12) that

$$
\left[\begin{array}{c}
\sqrt{\frac{d_{2}-d(t)}{\tau(t)-d_{1}}}\left(x\left(t-d_{1}\right)-x(t-\tau(t))\right) E \\
-\sqrt{\frac{d(t)-d_{1}}{d_{2}-\tau(t)}}\left(x(t-\tau(t))-x\left(t-d_{2}\right)\right) E
\end{array}\right]^{T}\left[\begin{array}{ll}
Z_{3} & R \\
R^{T} & Z_{3}
\end{array}\right]
$$$$
\left[\begin{array}{c}
\sqrt{\frac{d_{2}-\tau(t)}{\tau(t)-d_{1}}}\left(x\left(t-d_{1}\right)-x(t-\tau(t))\right) E \\
-\sqrt{\frac{\tau(t)-d_{1}}{d_{2}-\tau(t)}}\left(x(t-\tau(t))-x\left(t-d_{2}\right)\right) E
\end{array}\right] \geq 0 .
$$

Thus, according to Lemma 1, we obtain from (24) and (25) that

$$
\begin{aligned}
& -d_{21} \int_{t-d_{2}}^{t-d_{1}} \dot{x}^{T}(s) E^{T} Z_{3} E \dot{x}(s) d s \\
& \leq-\left[\begin{array}{c}
x\left(t-d_{1}\right)-x(t-\tau(t)) \\
x(t-\tau(t))-x\left(t-d_{2}\right)
\end{array}\right]^{T}\left[\begin{array}{cc}
E^{T} Z_{3} E & E^{T} R E \\
* & E^{T} Z_{3} E
\end{array}\right]\left[\begin{array}{c}
x\left(t-d_{1}\right)-x(t-\tau(t)) \\
x(t-\tau(t))-x\left(t-d_{2}\right)
\end{array}\right] \\
& =-\vartheta^{T}(t)\left[\begin{array}{ccc}
E^{T} Z_{3} E & -E^{T} Z_{3} E+E^{T} R E & -E^{T} R E \\
* & 2 E^{T} Z_{3} E-E^{T}\left(R^{T}+R\right) E & -E^{T} Z_{3} E+E^{T} R E \\
* & * & E^{T} Z_{3} E
\end{array}\right] \vartheta(t),
\end{aligned}
$$

where Substituting (26) into (22),

$$
\vartheta(t)=\left[\begin{array}{lll}
x^{T}\left(t-d_{1}\right) & x^{T}(t-\tau(t)) & x^{T}\left(t-d_{2}\right)
\end{array}\right]^{T} .
$$

$$
\dot{V}(t) \leq \varsigma^{T}(t)\left(\Phi+\left[\begin{array}{llll}
A & 0 & B K & 0
\end{array}\right]^{T}\left(d_{1}^{2} Z_{1}+d_{2}^{2} Z_{2}+d_{21}^{2} Z_{3}\right)\left[\begin{array}{llll}
A & 0 & B K & 0
\end{array}\right]\right) \varsigma(t),
$$

where 


$$
\begin{aligned}
\Phi & =\left[\begin{array}{cccc}
\Xi_{11} & E^{T} Z_{1} E & P^{T} B K & E^{T} Z_{2} E \\
* & \Xi_{22} & \Xi_{23} & E^{T} R E \\
* & * & \Xi_{33} & \Xi_{34} \\
* & * & * * & \Xi_{44}
\end{array}\right], \\
\zeta(t) & =\left[\begin{array}{lllll}
x^{T}(t) & x^{T}\left(t-d_{1}\right) & x^{T}(t-\tau(t)) & x^{T}\left(t-d_{2}\right)
\end{array}\right]^{T} .
\end{aligned}
$$

By the Schur complement, (28) guarantees that
Therefore, it can be obtained that $\dot{V}(t)<-\sigma\|x(t)\|^{2}$ when $x(t) \neq 0$ and $\sigma>0$. Then, system (9) is asymptotically stable.

Then, it will be proved that system (9) satisfies the $H_{\infty}$ performance index $\gamma$. Consider the $H_{\infty}$ performance for all nonzero $w(t) \in L_{2}[0, \infty)$ as follows:

$$
J_{z w} \leq \int_{0}^{\infty}\left[y^{T}(s) y(s)-\gamma^{2} w^{T}(s) w(s)\right] \mathrm{d} s, \gamma>0 .
$$

Then,

$\Phi+\left[\begin{array}{llll}A & 0 & B K & 0\end{array}\right]^{T}\left(d_{1}^{2} Z_{1}+d_{2}^{2} Z_{2}+d_{21}^{2} Z_{3}\right)\left[\begin{array}{llll}A & 0 & B K & 0\end{array}\right]<0$.

$$
\begin{aligned}
& y^{T}(t) y(t)-\gamma^{2} w^{T}(t) w(t)+\dot{V}(t) \\
& \leq \zeta^{T}(t)\left(\Theta+\left[\begin{array}{lllll}
C & 0 & D K & 0 & 0
\end{array}\right]^{T}\left[\begin{array}{lllll}
C & 0 & D K & 0 & 0
\end{array}\right]+\left[\begin{array}{ll}
A & 0
\end{array}\right.\right.
\end{aligned}
$$

$$
\left.\left.\begin{array}{ll}
0 & B_{w}
\end{array}\right]^{T}\left(d_{1}^{2} Z_{1}+d_{2}^{2} Z_{2}+d_{21}^{2} Z_{3}\right)\left[\begin{array}{lllll}
A & 0 & B K & 0 & B_{w}
\end{array}\right]\right) \zeta(t)
$$

where

$$
\begin{aligned}
\zeta(t) & =\left[\begin{array}{ccccc}
x^{T}(t) & x^{T}\left(t-d_{1}\right) & x^{T}(t-\tau(t)) & x^{T}\left(t-d_{2}\right) & w^{T}(t)
\end{array}\right]^{T}, \\
\Theta & =\left[\begin{array}{ccccc}
\Xi_{11} & E^{T} Z_{1} E & P^{T} B K & E^{T} Z_{2} E & P B_{w} \\
* & \Xi_{22} & \Xi_{23} & E^{T} R E & 0 \\
* & * & \Xi_{33} & \Xi_{34} & 0 \\
* & * & * & \Xi_{44} & 0 \\
* & * & * & * & -\gamma^{2} I
\end{array}\right] .
\end{aligned}
$$

$$
\begin{aligned}
\Theta & +\left[\begin{array}{llll}
C & 0 & D K & 0
\end{array}\right]^{T}\left[\begin{array}{llll}
C & 0 & D K & 0
\end{array}\right] \\
& +\left[\begin{array}{llll}
A & 0 & B K & B_{w}
\end{array}\right]^{T}\left(d_{1}^{2} Z_{1}+d_{2}^{2} Z_{2}+d_{21}^{2} Z_{3}\right)\left[\begin{array}{llll}
A & 0 & B K & B_{w}
\end{array}\right]<0 .
\end{aligned}
$$

From (34), we can obtain that

$$
y^{T}(t) y(t)-\gamma^{2} w^{T}(t) w(t)+\dot{V}(t)<0
$$

Under zero initial conditions, we have $V(0)=0$ and $V(\infty) \geq 0$. From (35), it can be obtained that $\|y(t)\|_{2} \leq \gamma\|w(t)\|_{2}$ for all nonzero $w(t) \in L_{2}[0, \infty)$.

Then, according to the condition, system (9) is asymptotically stable and satisfies the $H_{\infty}$ performance index $\gamma$. This completed the proof.

Remark 3. If $E=I, Q_{1}=Q_{2}=0$, and $R_{2}=R_{3}=0$, then the LKF is similar with the one in [31]. Therefore, the LKF in [31] is a special case of (9), which means that the result in Theorem 1 has wider application scopes than [31].

Remark 4. To reduce the conservativeness, severable methods have been reported, such as the free-weighting matrix method and Wirtinger-based inequality. In the constructed LKF, we directly use tighter interactive convex combinatorial inequalities to estimate the integral terms $-d_{21} \int_{t-d_{2}}^{t_{k}} \dot{x}^{T}(s) E^{T} Z_{3} E \dot{x}(s) \mathrm{d} s$.

During this process, a matrix $R$ is introduced to deal with the two terms

$$
\begin{aligned}
& \frac{d_{21}}{d_{2}-\tau(t)}\left(x(t-\tau(t))-x\left(t-d_{2}\right)\right)^{T} E^{T} Z_{3} E \\
& \cdot\left(x(t-\tau(t))-x\left(t-d_{2}\right)\right), \\
& \frac{d_{21}}{\tau(t)-d_{1}}\left(x\left(t-d_{1}\right)-x(t-\tau(t))\right)^{T} E^{T} Z_{3} E \\
& \cdot\left(x\left(t-d_{1}\right)-x(t-\tau(t))\right) .
\end{aligned}
$$


However, the above two terms are not considered in $[33,34]$, which will lead to conservatism to some extent. Besides, (25) is free as matrix $R$ could be more than nonnegative. Therefore, the conservatism has been greatly reduced.

Remark 5. It is noted that the number of decision variables is $4.5 n^{2}+4.5 n$, and it illustrates that the proposed methodology has lower computational complexity than the freeweighting matrix method or Jensen inequality lemma which increases the computational complexity with too much additional slack variables.

Furthermore, according to the following theorems, we will design the sampled-data controller to stabilize system (9).

Theorem 2. For scales $d_{1}, d_{2}$, and $\gamma$, system (9) is asymptotically admissible with $H_{\infty}$ performance $\gamma$ if there exist matrices $P, R, Q_{i}>0, Z_{i}>0, i=1,2,3$, satisfying

$$
\begin{gathered}
E^{T} P=P^{T} E \geq 0, \\
{\left[\begin{array}{cc}
\bar{Z}_{3} & \bar{R} \\
\bar{R}^{T} & \bar{Z}_{3}
\end{array}\right]>0,} \\
{\left[\begin{array}{cc}
\bar{\Pi}_{11} & \bar{\Pi}_{12} \\
* & \bar{\Pi}_{22}
\end{array}\right]<0,}
\end{gathered}
$$

where

$$
\begin{aligned}
& \Pi_{11}=\left[\begin{array}{ccccc}
\bar{\Xi}_{11} & E^{T} \bar{Z}_{1} E & B \bar{K} & E^{T} \bar{Z}_{2} E & B_{w} \\
* & \bar{\Xi}_{22} & \bar{\Xi}_{23} & E^{T} \bar{R} E & 0 \\
* & * & \bar{\Xi}_{33} & \bar{\Xi}_{34} & 0 \\
* & * & * & \bar{\Xi}_{44} & 0 \\
* & * & * & * * & -\gamma^{2} I
\end{array}\right], \\
& \Pi_{12}=\left[\begin{array}{ccccc}
C \bar{P}^{T} & 0 & \overline{D K} & 0 & 0 \\
A \bar{P}^{T} & 0 & \overline{B K} & 0 & B_{w} \bar{P}^{T}
\end{array}\right]^{T}, \\
& \Pi_{22}=\operatorname{diag}\left\{-I,-\bar{P} Z^{-1} \bar{P}\right\} \text {, } \\
& \bar{\Xi}_{11}=A \bar{P}+\bar{P} A^{T}+\bar{Q}_{1}+\bar{Q}_{2}+\bar{Q}_{3}-E^{T} \bar{Z}_{1} E-E^{T} \bar{Z}_{2} E, \\
& \bar{\Xi}_{22}=-\bar{Q}_{1}-E^{T} \bar{Z}_{1} E-E^{T} \bar{Z}_{3} E \text {, } \\
& \bar{\Xi}_{23}=E^{T} \bar{Z}_{3} E-E^{T} \bar{R} E \text {, } \\
& \Xi_{33}=-2 E^{T} \bar{Z}_{3} E+E^{T} \bar{R} E+E^{T} \bar{R}^{T} E, \\
& \bar{\Xi}_{34}=E^{T} \bar{Z}_{3} E-E^{T} \bar{R} E \text {, } \\
& \Xi_{44}=-\bar{Q}_{3}-E^{T} \bar{Z}_{2} E-E^{T} \bar{Z}_{3} E, \\
& \bar{Z}=d_{1}^{2} \bar{Z}_{1}+d_{2}^{2} \bar{Z}_{2}+d_{21}^{2} \bar{Z}_{3} \text {, } \\
& d_{21}=d_{2}-d_{1} \text {. }
\end{aligned}
$$

Then, the controller gain matrix $K$ can be obtained such that

$$
K=\overline{K P}^{-1} \text {. }
$$

Proof. By noticing that $-\bar{P} \bar{Z}^{-1} \bar{P} \leq \bar{Z}-2 \bar{P}$ let $\eta=\operatorname{diag}\left\{P^{-T}, P^{-T}, P^{-T}, P^{-T}, I, I, I\right\}$ and,$\quad \kappa=\operatorname{diag}\left\{P^{-T}\right.$, $\left.P^{-T}\right\}$, and define

$$
\begin{aligned}
\bar{P} & =P^{-1}, \\
\bar{K} & =K P^{-1}, \\
\bar{Z} & =P^{-T} Z P^{-1}, \\
\bar{R} & =P^{-T} R P^{-1}, \\
\bar{Z}_{i} & =P^{-T} Z_{i} P^{-1}, \\
\bar{Q}_{i} & =P^{-T} Q_{i} P^{-1}, i=1,2,3 .
\end{aligned}
$$

Pre- and postmultiplying (12) by $\kappa$ and $\kappa^{T}$, respectively, we can obtain (33). Pre- and postmultiplying (13) by $\eta$ and $\eta^{T}$, respectively, (34) can be obtained by the Schur complement. The proof is completed.

Remark 6. It can be seen that the control performance is affected by the design parameters $d_{1}, d_{2}$, and these parameters are chosen to solve the LMI problem. We can adjust the design parameters to achieve a better solution. The design and implementation step of the proposed method is provided as follows.

Step 1. We solve the LMI problem $P$ s to guarantee the system is admissible.

$$
P s:\left\{\begin{array}{l}
\text { minimize } \gamma \\
\text { subject to } P, R, Q_{i}>0, Z_{i}>0, i=1,2,3 \\
\text { LMIs (11), (12), (13). }
\end{array}\right.
$$

Step 2. If the above problem is feasible, then we solve the LMI problems $P o$ to obtain the sampled-data controller. Otherwise, we adjust the design parameters $d_{1}, d_{2}$, and then go back to Step 1.

$$
\text { Po: }\left\{\begin{array}{l}
\operatorname{minimize} \gamma, \\
\text { subject to } \bar{P}, \bar{R}, \bar{Q}_{i}>0, \bar{Z}_{i}>0, \quad i=1,2,3, \\
\operatorname{LMIs}(30),(31),(32) .
\end{array}\right.
$$

Step 3. If the above problem is solvable, we can obtain the controller, that is, $K=\overline{K P}^{-1}$. Otherwise, we adjust the design parameters $d_{1}, d_{2}$, and then go back to Step 2 .

The flowchart of the proposed method is provided in Figure 2.

Then, when $E=I$, the following Corollary 1 can be obtained based on Theorem 2. According to Corollary 1, we can use the proposed method to solve the sampled-data control problem of the practical systems, such as dynamic positioning ship and autonomous airship.

Corollary 1. For scales $d_{1}, d_{2}$, and $\gamma$, system (9) is asymptotically stable with $H_{\infty}$ performance $\gamma$ if there exist matrices $P, R, Q_{i}>0, Z_{i}>0, i=1,2,3$, such that 


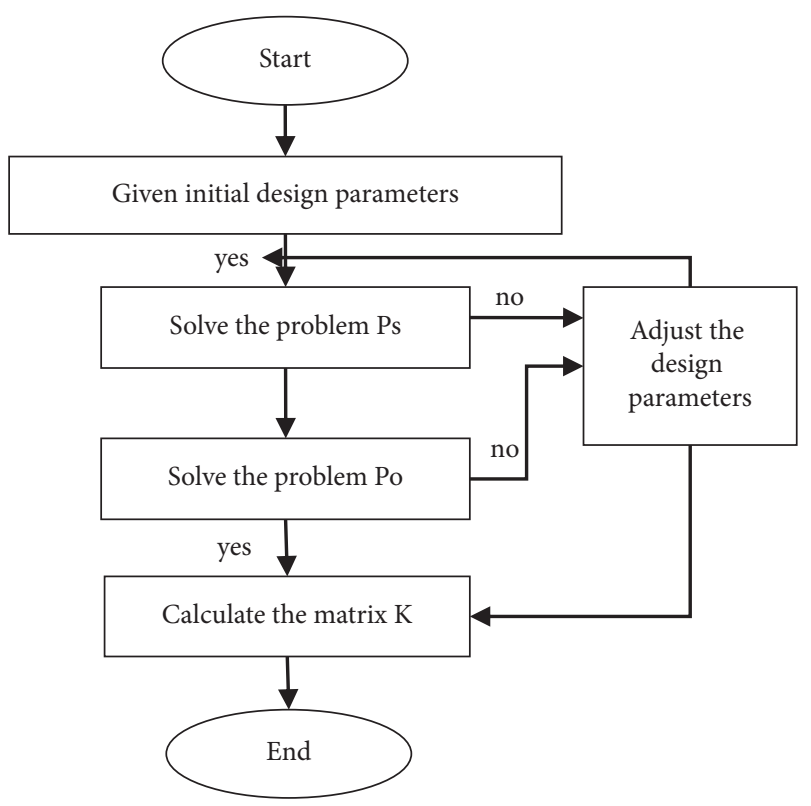

FIgURE 2: The flowchart of the proposed method.

$$
\begin{aligned}
& {\left[\begin{array}{cc}
\bar{Z}_{3} & \bar{R} \\
\bar{R}^{T} & \bar{Z}_{3}
\end{array}\right]>0,} \\
& {\left[\begin{array}{cc}
\bar{\Pi}_{11} & \bar{\Pi}_{12} \\
* & \bar{\Pi}_{22}
\end{array}\right]<0,}
\end{aligned}
$$

where

$$
\begin{aligned}
& \Pi_{11}=\left[\begin{array}{ccccc}
\bar{\Xi}_{11} & \bar{Z}_{1} & B \bar{K} & \bar{Z}_{2} & B_{w} \\
* & \bar{\Xi}_{22} & \bar{\Xi}_{23} & \bar{R} & 0 \\
* & * & \bar{\Xi}_{33} & \bar{\Xi}_{34} & 0 \\
* & * & * & \bar{\Xi}_{44} & 0 \\
* & * & * & * & -\gamma^{2} I
\end{array}\right], \\
& \Pi_{12}=\left[\begin{array}{ccccc}
C \bar{P}^{T} & 0 & \overline{D K} & 0 & 0 \\
A \bar{P}^{T} & 0 & \overline{B K} & 0 & B_{w} \bar{P}^{T}
\end{array}\right]^{T}, \\
& \Pi_{22}=\operatorname{diag}\left\{-I,-\bar{P} Z^{-1} \bar{P}\right\} \text {, } \\
& \bar{\Xi}_{11}=A \bar{P}+\bar{P} A^{T}+\bar{Q}_{1}+\bar{Q}_{2}+\bar{Q}_{3}-\bar{Z}_{1}-\bar{Z}_{2} \text {, } \\
& \bar{\Xi}_{22}=-\bar{Q}_{1}-\bar{Z}_{1}-\bar{Z}_{3} \text {, } \\
& \bar{\Xi}_{23}=\bar{Z}_{3}-\bar{R} \text {, } \\
& \Xi_{33}=-2 \bar{Z}_{3}+\bar{R}+\bar{R}^{T} \text {, } \\
& \bar{\Xi}_{34}=\bar{Z}_{3}-\bar{R} \text {, } \\
& \Xi_{44}=-\bar{Q}_{3}-\bar{Z}_{2}-\bar{Z}_{3} \text {, } \\
& \bar{Z}=d_{1}^{2} \bar{Z}_{1}+d_{2}^{2} \bar{Z}_{2}+d_{21}^{2} \bar{Z}_{3} \text {, } \\
& d_{21}=d_{2}-d_{1} \text {. }
\end{aligned}
$$
that

Then, the controller gain matrix $K$ can be obtained such

$$
K=\overline{K P}^{-1}
$$

Proof. The procedure of the proof is similar with Theorem 2, so it is omitted.

\section{Numerical Examples}

In this section, to validate the effectiveness of the given method, two numerical examples are introduced.

Example 1. Consider the following parameters:

$$
\begin{aligned}
A & =\left[\begin{array}{rr}
-13.1 & -13.7 \\
-15.4 & -23.8
\end{array}\right], \\
B & =\left[\begin{array}{rr}
-18.6 & -10.4 \\
-25.2 & -16.8
\end{array}\right], \\
B_{w} & =\left[\begin{array}{l}
1.9 \\
1.8
\end{array}\right], \\
C & =\left[\begin{array}{ll}
0.4 & -0.8
\end{array}\right], \\
E & =\left[\begin{array}{ll}
9 & 3 \\
6 & 2
\end{array}\right], \\
D & =\left[\begin{array}{ll}
1 & 1
\end{array}\right] .
\end{aligned}
$$

When $w(t)=0$, for different lower bounds of the time delay $d_{1}$, Table 1 lists the maximum time delay $d_{2}$ to guarantee the admissibility of the system by different methods. From Table 1, the upper bound of time delay $d_{2}$ obtained by Theorem 1 is larger than that in [33-36], which indicates that the proposed method in the paper can obtain lower conservative results than the other references.

Next, consider the situation about $w(t) \neq 0$. For different $d_{2}>0$, Table 2 lists the minimal $H_{\infty}$ performance indicators $\gamma$ that guarantee the admissibility of the system by Theorem 1 and [37] when $d_{1}=0$. From Table 2, it is easy to see that, for different upper bounds of time delay $d_{2}, H_{\infty}$ performance $\gamma$ obtained by Theorem 1 is smaller than that in [37]. Therefore, it is shown that Theorem 1 improves the results of [37].

Assuming the sampling interval $d_{2}=1.7$, the $H_{\infty}$ performance is obtained such that $\gamma_{\min }=0.8625$. Then, the controller gain can be computed such that

$$
K=\left[\begin{array}{ll}
-0.1663 & -0.1109 \\
-0.0554 & -0.0370
\end{array}\right] \text {. }
$$

Consider the system's initial state $x_{s}(t)=\left[\begin{array}{ll}-2 & -2\end{array}\right]$ and the external environment disturbance $w(t)=0.5 \cos (t)$ and random disturbance, respectively. Under different disturbances, the response curves of the system's state and the control input $u(t)$ are shown in Figures 3-6, respectively. From Figures 3 and 5, it is shown that the system's state $x_{1}(t)$ and $x_{2}(t)$ are stable in a short time under different disturbances, which further illustrates that the designed sampled-data controller can make the system's states stable and have acceptable control performance with external variable disturbances. 
TABLE 1: Maximum values of the upper bound $d_{2}$.

\begin{tabular}{lccccc}
\hline$d_{1}$ & 1.4 & 1.6 & 1.8 & 2.0 & 2.2 \\
{$[33]$} & 2.1121 & 2.1450 & 2.2841 & 2.4328 & 2.5852 \\
{$[34]$} & 2.2314 & 2.2761 & 2.4041 & 2.5383 & 2.6777 \\
{$[35]$} & 2.3360 & 2.3704 & 2.4242 & 2.5425 & 2.7007 \\
{$[36]$} & 2.3372 & 2.3730 & 2.4923 & 2.6181 & 2.7494 \\
Theorem 1 & 2.3418 & 2.3836 & 2.5013 & 2.6408 & 2.7826 \\
\hline
\end{tabular}

TABLe 2: Minimum values of $H_{\infty}$ performance $\gamma\left(d_{1}=0\right)$.

\begin{tabular}{lccccc}
\hline$d_{2}$ & 0.9 & 1.1 & 1.3 & 1.5 & 1.7 \\
[37] & 0.2713 & 0.3548 & 0.4744 & 0.6378 & 0.8736 \\
Theorem 1 & 0.2627 & 0.3468 & 0.4642 & 0.6174 & 0.8625 \\
\hline
\end{tabular}

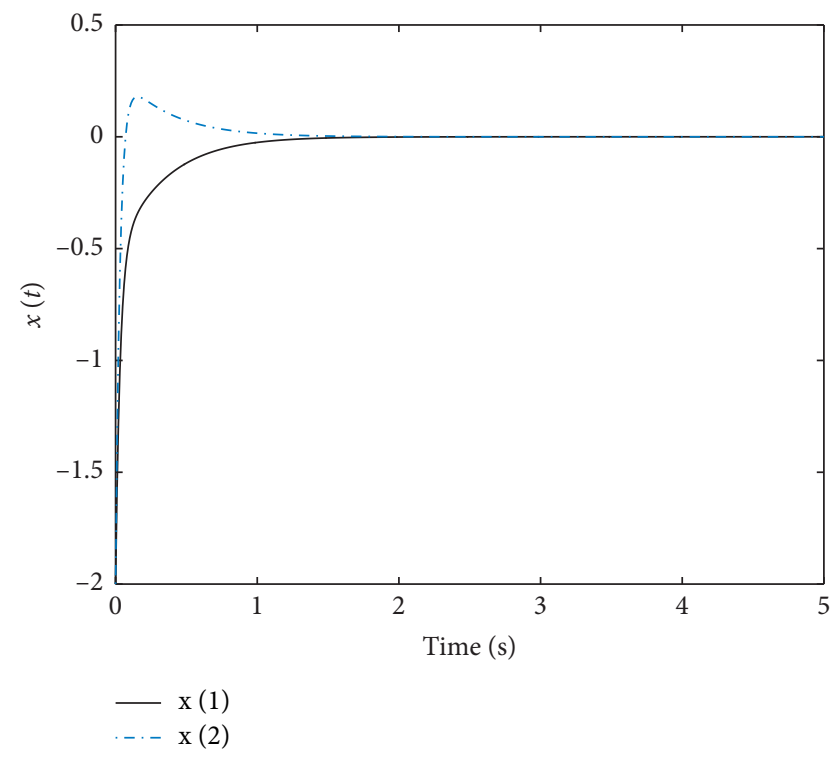

Figure 3: The state response curve of system (9) $(w(t)=0.5 \cos (t))$.

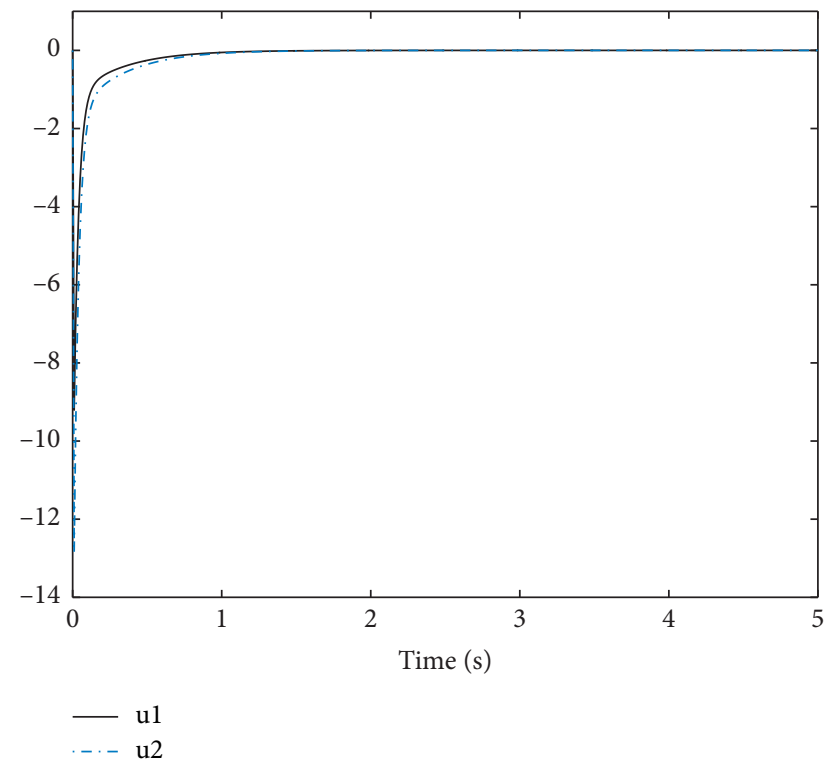

Figure 4: Control input for system (9) $(w(t)=0.5 \cos (t))$.

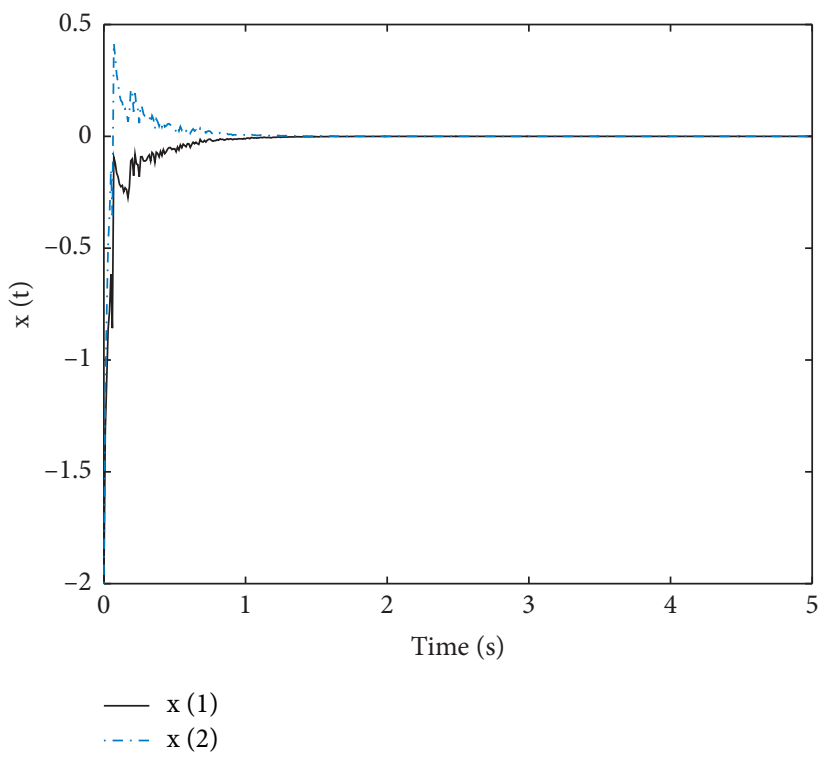

Figure 5: The state response curve of system $(9)(w(t)$ is the random disturbance).

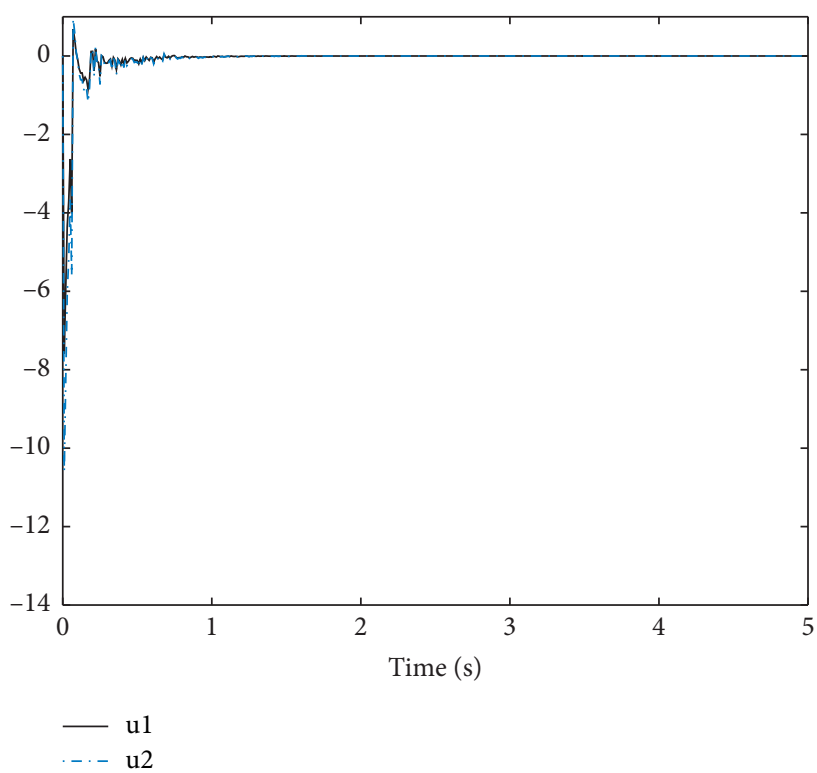

FIgure 6: Control input for system (9) $(w(t)$ is the random disturbance).

Example 2. Similar with [38], the motion equations of a sampled-data dynamic positioning ship system are considered as follows:

$$
M \dot{v}+D v=\tau+w
$$

where $\tau$ denotes the control input, $w$ denotes the external disturbance, $M$ is the inertia matrix, $D$ is the damping coefficient matrix, and $v=[p, v, r]^{T}$ includes the velocity of surge, sway, and yaw, respectively. Define the input matrix

$$
x(t)=\left[x_{a}, y_{a}, \psi, p, v, r\right]^{T},
$$




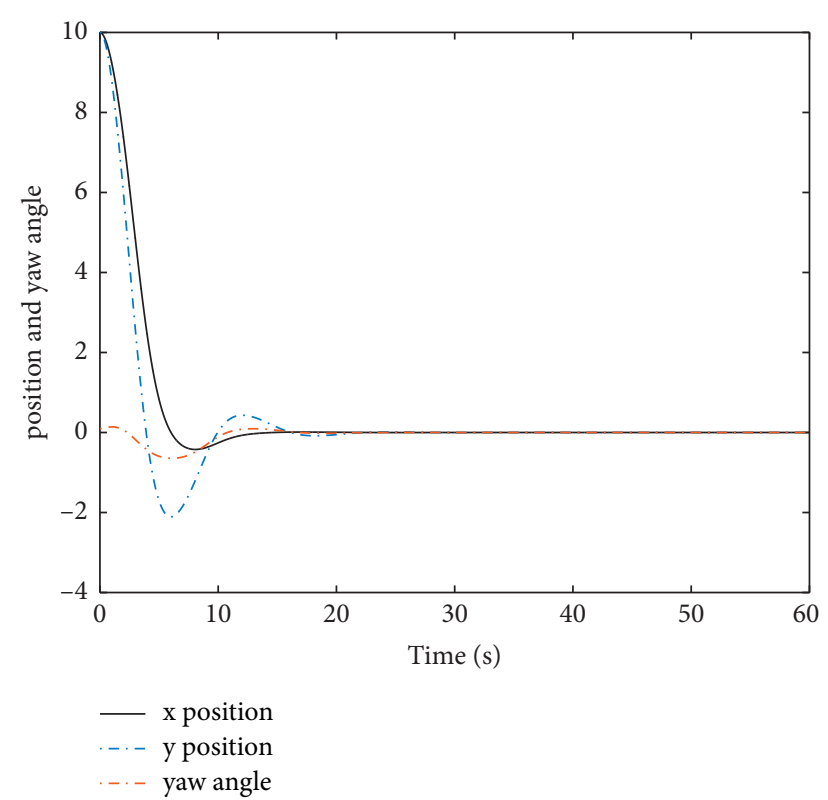

FIGURE 7: The responses of the position of $x$ and $y$ and yaw angle of DPS.

where $x_{a}, y_{a}$, and $\psi$ represent the $x$-position, $y$-position, and yaw angle, respectively. The values of $M$ and $D$ are considered such that

$$
\begin{aligned}
M & =\left[\begin{array}{ccc}
0.754 & 0 & 0 \\
0 & 1.199 & 0.211 \\
0 & 0.029 & 0.524
\end{array}\right], \\
D & =\left[\begin{array}{ccc}
0.014 & 0 & 0 \\
0 & 0.102 & -0.024 \\
0 & 0.192 & 0.095
\end{array}\right] .
\end{aligned}
$$

Let $A=\left[\begin{array}{cc}0 & I \\ 0-M^{-1} D\end{array}\right], B=\left[\begin{array}{c}0 \\ M^{-1}\end{array}\right]$, and $B_{w}=\left[\begin{array}{c}0 \\ M^{-1}\end{array}\right]$.

Then,

$$
\begin{aligned}
& A=\left[\begin{array}{cccccc}
0 & 0 & 0 & 1 & -0.0349 & 0 \\
0 & 0 & 0 & 0.0349 & 1 & 0 \\
0 & 0 & 0 & 0 & 0 & 1 \\
0 & 0 & 0 & -0.0186 & 0 & 0 \\
0 & 0 & 0 & 0 & -0.0208 & 0.0342 \\
0 & 0 & 0 & 0 & -0.3653 & -0.1832
\end{array}\right], \\
& B=B_{w} \\
& =\left[\begin{array}{ccc}
0 & 0 & 0 \\
0 & 0 & 0 \\
0 & 0 & 0 \\
1.3263 & 0 & 0 \\
0 & 0.8422 & -0.3391 \\
0 & -0.0466 & 1.9272
\end{array}\right] .
\end{aligned}
$$

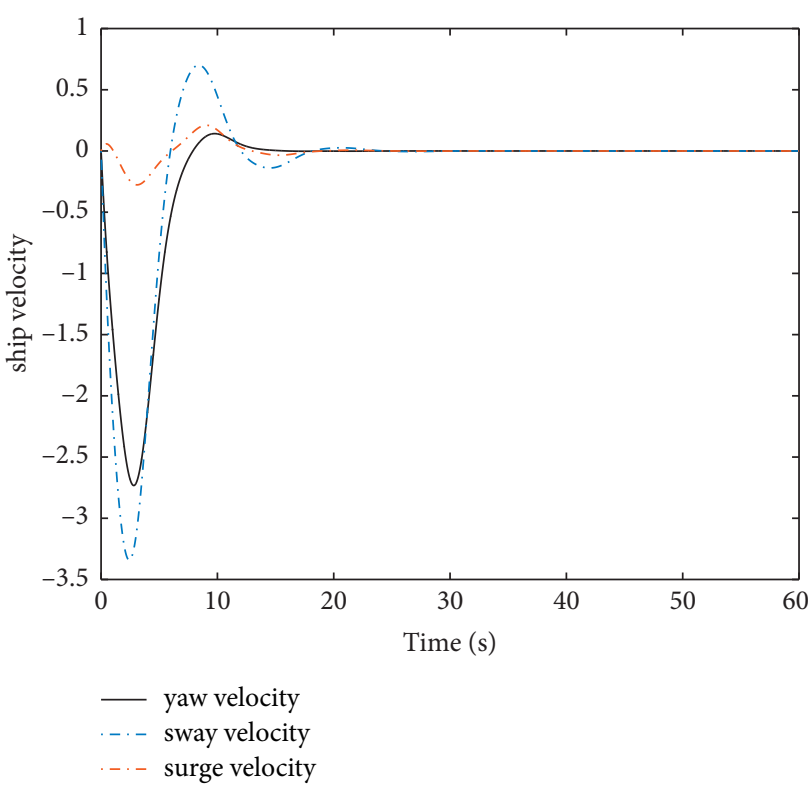

FIgURE 8: The response of velocities of DPS.

The initial states of the ship are assumed such that $x_{s}(t)=\left[\begin{array}{llllll}10 & 10 & 0.1 & 0 & 0 & 0\end{array}\right]^{T}$. Let $w(t)=\sin (0.1 t)$. And the values of the sampling period are $d_{1}=0.8$ and $d_{2}=1.6$; then, the $H_{\infty}$ performance index is obtained according to Corollary 1 such that $\gamma_{\min }=2.945$. Then, the controller gain is obtained as follows:

$K=\left[\begin{array}{cccccc}-0.2438 & -0.0109 & -0.0008 & -0.5459 & -0.0105 & 0.0002 \\ 0.0072 & -0.4429 & -0.1018 & 0.0186 & -0.5794 & -0.1722 \\ -0.0011 & 0.0088 & -0.2082 & -0.0022 & 0.2673 & -0.5638\end{array}\right]$.

The responses of the DPS state are shown in Figures 7 and 8 . Figure 7 shows the responses of the position of $x$ and $y$ and yaw angle of DPS. Figure 8 shows the response of velocities of DPS. It is shown that the DPS can be stabilized by the designed sampled-data controller even if external disturbances exist.

\section{Conclusion}

The sampled-data control issue for singular systems is discussed in this paper. Both lower and upper bounds of the variable period have been considered. Then, the criteria of asymptotical admissibility are given by constructing a suitable LKF. Then, the tighter reciprocally convex inequalities are used to estimate the derivative of the LKF, and less conservative results can be obtained. Two numerical examples are given to illustrate the effectiveness of the proposed method. In the future, we will improve the proposed methods and consider the actuator faults and then discuss the sampled-data fault-tolerant issue for the more complex nonlinear singular systems such as fuzzy singular systems and singular Markov jump systems. 


\section{Conflicts of Interest}

The authors declare that they have no conflicts of interest.

\section{Acknowledgments}

This work was supported by the National Natural Science Foundation of China (51579114 and 51879119), the Natural Science Foundation of Fujian Province (2018J01484 and 2021J01822), the Project of Young and Middle-aged Teacher Education of Fujian Province (JAT170311), Scholarship of studying abroad in the Education Department of Fujian Province, and the Youth Innovation Foundation of Xiamen (3502Z20206019).

\section{References}

[1] Y. Wang, H. R. Karimi, H. Shen, Z. Fang, and M. Liu, "Fuzzymodel-based sliding mode control of nonlinear descriptor systems," IEEE Transactions on Cybernetics, vol. 99, pp. 1-11, 2018.

[2] T. Xu, Q. Zhang, and F. Zhao, "Ho filtering problem of singular systems with uncertainties in the difference matrix," International Journal of Control, Automation and Systems, vol. 16, no. 3, pp. 1207-1216, 2018.

[3] S. Zheng, Y. Zhou, S. Yang, and L. Li, "Projective synchronization analysis of drive-response coupled dynamical network with multiple time-varying delays via impulsive control," Abstract and Applied Analysis, vol. 2014, pp. 1-10, 2014.

[4] M. Zheng, Y. Zhou, S. Yang, L. Li, and Y. Suo, "Ho sampleddata control for singular neutral system," Mathematical Problems in Engineering, vol. 2017, pp. 1-16, 2017.

[5] Y. Wang, Y. Gao, H. R. Karimi, H. Shen, and Z. Fang, "Notice of violation of IEEE publication principles: sliding mode control of fuzzy singularly perturbed systems with application to electric circuit," IEEE Transactions on Systems, Man, and Cybernetics: Systems, vol. 48, no. 10, pp. 1667-1675, 2018.

[6] M. Zheng, Y. Zhou, S. Yang, and L. Li, "Sampled-data control for nonlinear singular systems based on a Takagi-Sugeno fuzzy model," Transactions of the Institute of Measurement and Control, vol. 40, no. 14, pp. 4027-4036, 2018.

[7] T. Taniguchi, K. Tanaka, K. Yamafuji, and H. O. Wang, "Fuzzy descriptor systems: stability analysis and design via LMIs,"vol. 3, pp. 1827-1831, in Proceedings of the 1999 American Control Conference, vol. 3, IEEE, San Diego, CA, USA, June 1999.

[8] B. Meng and J. F. Zhang, "Output feedback based admissible control of switched linear singular systems," Acta Automatica Sinica, vol. 32, no. 2, pp. 179-185, 2006.

[9] X.-z. Dong, "Admissibility analysis of linear singular systems via a delta operator method," International Journal of Systems Science, vol. 45, no. 11, pp. 2366-2375, 2014.

[10] S. Xing, Q. Zhang, and B. Zhu, "Mean-square admissibility for stochastic T-S fuzzy singular systems based on extended quadratic Lyapunov function approach," Fuzzy Sets and Systems, vol. 307, pp. 99-114, 2017.

[11] M. Chadli, H. R. Karimi, and P. Shi, "On stability and stabilization of singular uncertain Takagi-Sugeno fuzzy systems," Journal of the Franklin Institute, vol. 351, no. 3, pp. 1453-1463, 2014.

[12] Y. Wang, X. Yang, and H. Yan, "Reliable fuzzy tracking control of near-space hypersonic vehicle using aperiodic measurement information," IEEE Transactions on Industrial Electronics, vol. 66, no. 12, pp. 9439-9447, 2019.

[13] S. M. Abedi, "An optimal sample-data holds by using a Biobjective criterion: trade-off between the phase delay and the stability robustness," Control Engineering and Applied Informatics, vol. 17, no. 2, pp. 32-42, 2015.

[14] Y. Wang, H. R. Karimi, H.-K. Lam, and H. Shen, "Notice of violation of IEEE publication principles: an improved result on exponential stabilization of sampled-data fuzzy systems," IEEE Transactions on Fuzzy Systems, vol. 26, no. 6, pp. 3875-3883, 2018.

[15] Y. Wang, H. Pu, P. Shi, C. K. Ahn, and J. Luo, "Sliding mode control for singularly perturbed Markov jump descriptor systems with nonlinear perturbation," Automatica, vol. 127, Article ID 109515, 2021.

[16] E. Fridman, "Robust sampled-data\$H_infty\$Control of linear singularly perturbed systems," IEEE Transactions on Automatic Control, vol. 51, no. 3, pp. 470-475, 2006.

[17] M. Moarref and L. Rodrigues, "Sensor allocation with guaranteed exponential stability for linear multi-rate sampled-data systems," International Journal of Robust and Nonlinear Control, vol. 26, no. 7, pp. 1512-1529, 2016.

[18] Z. G. Wu, P. Shi, and H. Y. Su, "Stochastic synchronization of markovian jump neural networks with time-varying delay using sampled data," IEEE Transactions on Cybernetics, vol. 43, no. 6, pp. 796-1806, 2013.

[19] Y. Wang, X. Xie, M. Chadli, S. Xie, and Y. Peng, "Sliding mode control of fuzzy singularly perturbed descriptor systems," IEEE Transactions On Fuzzy Systems, vol. 29, 2020.

[20] Y. Wang, W. Zhou, J. Luo, H. Yan, H. Pu, and Y. Peng, "Reliable Intelligent Path Following Control for a Robotic Airship against Sensor Faults," IEEE/ASME Transactions on Mechatronics, vol. 24, 2019.

[21] F. Feng Ding and T. Tongwen Chen, "Hierarchical identification of lifted state-space models for general dual-rate systems," IEEE Transactions on Circuits and Systems I: Regular Papers, vol. 52, no. 6, pp. 1179-1187, 2005.

[22] L.-S. Hu, P. Shi, and P. M. Frank, "Robust sampled-data control for Markovian jump linear systems," Automatica, vol. 42, no. 11, pp. 2025-2030, 2006.

[23] K. Liu and E. Fridman, "Wirtinger's inequality and Lyapunovbased sampled-data stabilization," Automatica, vol. 48, no. 1 , pp. 102-108, 2012.

[24] K. Hooshmandi, F. Bayat, M. R. Jahed-Motlagh, and A. A. Jalali, "Stability analysis and design of nonlinear sampled-data systems under aperiodic samplings," International Journal of Robust and Nonlinear Control, vol. 28, no. 7, pp. 2679-2699, 2018.

[25] K. Hooshmandi, F. Bayat, M. R. Jahed-Motlagh, and A. Jalali, "Robust sampled-data control of non-linear LPV systems: time-dependent functional approach," IET Control Theory \& Applications, vol. 12, no. 9, pp. 1318-1331, 2018.

[26] S. Li, L. Yang, K. Li, and Z. Gao, "Robust sampled-data cruise control scheduling of high speed train," Transportation Research Part C: Emerging Technologies, vol. 46, pp. 274-283, 2014.

[27] Y.-y. Wang, Q.-b. Wang, P.-f. Zhou, and D.-p. Duan, "Robust $\mathrm{H} \infty$ directional control for a sampled-data autonomous airship," Journal of Central South University, vol. 21, no. 4, pp. 1339-1346, 2014.

[28] M. Zheng, Y. Zhou, S. Yang, and L. Li, "Robust Ho control of neutral system for sampled-data dynamic positioning ships," IMA Journal of Mathematical Control and Information, vol. 36, no. 4, pp. 1325-1345, 2019. 
[29] L. Dai, "Singular Control Systems," Lecture Notes in Control and Information Sciences, Vol. 118, Springer, , Manhattan, NY, USA, 1989.

[30] S. Shengyuan Xu, P. Van Dooren, R. Stefan, and J. Lam, "Robust stability and stabilization for singular systems with state delay and parameter uncertainty," IEEE Transactions on Automatic Control, vol. 47, no. 7, pp. 1122-1128, 2002.

[31] J. Yoneyama, "Robust sampled-data stabilization of uncertain fuzzy systems via input delay approach," Information Sciences, vol. 198, pp. 169-176, 2012.

[32] P. Park, J. W. Ko, and C. Jeong, "Reciprocally convex approach to stability of systems with time-varying delays," Automatica, vol. 47, no. 1, pp. 235-238, 2011.

[33] Z. Wu, H. Su, and J. Chu, "H $\infty$ filtering for singular systems with time-varying delay," International Journal of Robust and Nonlinear Control, vol. 20, no. 11, pp. 1269-1284, 2010.

[34] A. Haidar and E. K. Boukas, "Exponential stability of singular systems with multiple time-varying delays," Automatica, vol. 45, no. 2, pp. 539-545, 2009.

[35] X. Zhu, Y. Wang, and Y. Gan, "Ho filtering for continuoustime singular systems with time-varying delay," International Journal of Adaptive Control and Signal Processing, vol. 25, no. 2, pp. 137-154, 2011.

[36] Z.-G. Wu, J. H. Park, H. Su, and J. Chu, "Dissipativity analysis for singular systems with time-varying delays," Applied Mathematics and Computation, vol. 218, no. 8, pp. 4605-4613, 2011.

[37] Y. S. Lee, Y. S. Moon, W. H. Kwon, and P. G. Park, "Delaydependent robust $\mathrm{H} \infty$ control for uncertain systems with a state-delay," Automatica, vol. 40, no. 1, pp. 65-72, 2004.

[38] A. J. Sorensen, "A survey of dynamic positioning control systems," Annual Reviews in Control, vol. 35, no. 1, pp. 123-136, 2011. 